TRANSACTIONS OF THE

AMERICAN MATHEMATICAL SOCIETY

Volume 359, Number 11, November 2007, Pages 5653-5668

S 0002-9947(07)04336-X

Article electronically published on May 8, 2007

\title{
SINGULAR SOLUTIONS OF PARABOLIC $p$-LAPLACIAN WITH ABSORPTION
}

\author{
XINFU CHEN, YUANWEI QI, AND MINGXIN WANG
}

\begin{abstract}
We consider, for $p \in(1,2)$ and $q>1$, the $p$-Laplacian evolution equation with absorption

$$
u_{t}=\operatorname{div}\left(|\nabla u|^{p-2} \nabla u\right)-u^{q} \text { in } \mathbb{R}^{n} \times(0, \infty) .
$$

We are interested in those solutions, which we call singular solutions, that are non-negative, non-trivial, continuous in $\mathbb{R}^{n} \times[0, \infty) \backslash\{(0,0)\}$, and satisfy $u(x, 0)=0$ for all $x \neq 0$. We prove the following:

(i) When $q \geq p-1+p / n$, there does not exist any such singular solution.

(ii) When $q<p-1+p / n$, there exists, for every $c>0$, a unique singular solution $u=u_{c}$ that satisfies $\int_{\mathbb{R}^{n}} u(\cdot, t) \rightarrow c$ as $t \searrow 0$.

Also, $u_{c} \nearrow u_{\infty}$ as $c \nearrow \infty$, where $u_{\infty}$ is a singular solution that satisfies $\int_{\mathbb{R}^{n}} u_{\infty}(\cdot, t) \rightarrow \infty$ as $t \searrow 0$.

Furthermore, any singular solution is either $u_{\infty}$ or $u_{c}$ for some finite positive c.
\end{abstract}

\section{INTRODUCTION}

We are interested in singular solutions for the parabolic $p$-Laplacian equation with absorption:

$$
u_{t}=\operatorname{div}\left(|\nabla u|^{p-2} \nabla u\right)-u^{q} \quad \text { in } \mathbb{R}^{n} \times(0, \infty) .
$$

Here by a singular solution we always mean a non-negative and non-trivial solution which is continuous in $\mathbb{R}^{n} \times[0, \infty) \backslash\{(0,0)\}$ and satisfies

$$
\lim _{t \searrow 0} \sup _{|x|>\varepsilon} u(x, t)=0 \quad \forall \varepsilon>0 .
$$

A singular solution is called a fundamental solution (FS for short) with mass $c \in$ $(0, \infty)$ if

$$
\lim _{t \searrow 0} \int_{|x|<1} u(x, t) d x=c .
$$

A singular solution is called a very singular solution (VSS for short) if

$$
\lim _{t \searrow 0} \int_{|x|<1} v(x, t) d x=\infty .
$$

Received by the editors May 7, 2002 and, in revised form, May 15, 2006.

2000 Mathematics Subject Classification. Primary 35K65, 35K15.

Key words and phrases. $p$-Laplacian, fast diffusion, absorption, fundamental solution, very singular solution.

All the authors are grateful to the Hong Kong RGC Grant HKUST 630/95P given to the second author. The first author would like to thank the National Science Foundation for Grant DMS-9971043, USA. The third author thanks the PRC for NSF Grant NSFC-19831060.

(C)2007 American Mathematical Society Reverts to public domain 28 years from publication 5653 
As one can see from the next section, a singular solution is either an FS or a VSS.

Denote by $\delta(\cdot)$ the Dirac delta function. Then (1.2) and (1.3) can be written in short as $u(\cdot, 0)=c \delta(\cdot)$. Typical diffusion equations without absorption, such as the heat equation $u_{t}=\Delta u$, the porous media equation $u_{t}=\Delta u^{m}$, and the parabolic $p$-Laplacian equation $u_{t}=\operatorname{div}\left(|\nabla u|^{p-2} \nabla u\right)$, admit only FSs; cf. [12] .

One observes that (1.1) is invariant under any rotation of $x$ and the scaling $u \rightarrow \mathcal{T}^{\lambda}(u)$ for any $\lambda>0$, where

$$
\mathcal{T}^{\lambda}(u)(x, t):=\lambda^{\alpha} u\left(\lambda^{\alpha \beta} x, \lambda t\right), \quad \alpha=\frac{1}{q-1}, \quad \beta=\frac{q+1-p}{p} .
$$

We call a singular solution of (1.1) self-similar if it is invariant under any rotation of $x$ and under the scaling $u \rightarrow \mathcal{T}^{\lambda}(u)$ for any $\lambda>0$, so that it necessarily takes the form $u=t^{-\alpha} w\left(|x| t^{-\alpha \beta}\right)$, where $w$ is defined on $[0, \infty)$ and satisfies the following ODE:

$$
\left\{\begin{array}{l}
\left(\left|w^{\prime}\right|^{p-2} w^{\prime}\right)^{\prime}+(n-1)\left|w^{\prime}\right|^{p-2} w^{\prime} / r+\alpha\left(\beta r w^{\prime}+w\right)-w^{q}=0 \quad \forall r>0, \\
w(r) \geq 0 \text { in }[0, \infty), \quad w(0)<\infty, \\
\lim _{r \rightarrow \infty} r^{1 / \beta} w(r)=0,
\end{array}\right.
$$

where the last condition is equivalent to (1.2) since, for $r=|x| t^{-\alpha \beta}, u(x, t)=$ $|x|^{-1 / \beta} r^{1 / \beta} w(r)$.

Singular solutions, as can be seen from the standard heat equation $u_{t}-\Delta u=0$, play a vital role in understanding the large time asymptotic behavior of solutions of the Cauchy problem of (1.1) with "fast" decaying initial data; see [7, 8, 10, 13, 24] and the references therein for some of their applications.

When $p>2$, (1.1) is degenerate parabolic. Using an ODE shooting method, Peletier and Wang [22] proved that when $q \in(p-1, p-1+p / n)$, (1.1) admits a self-similar VSS. Uniqueness of such a self-similar VSS was later verified by Diaz and Saa [5].

A complete investigation for all singular solutions of (1.1) for $p>2$ was performed by Kamin and Vazquez [13]. They proved the following:

(1) when $q \geq p-1+p / n$, (1.1) does not have any singular solution;

(2) when $p-1<q<p-1+p / n$, there exist a unique VSS and, for every given $c>0$, a unique FS with initial mass $c$;

(3) when $0<q \leq p-1$, there does not exist any VSS but there exists, for any $c>0$, a unique FS with initial mass $c$.

Actually, they provided the above classification for the following more general equation:

$$
u_{t}=\operatorname{div}\left(|\nabla u|^{p-2} \nabla u\right)-\phi(u) \quad \text { in } \mathbb{R}^{n} \times(0, \infty),
$$

where $\phi(\cdot)$ is in a certain class of non-negative functions that mimic $u^{q}$.

When $p=2$, equation (1.1) becomes a semilinear heat equation

$$
u_{t}=\Delta u-u^{q}, \quad q>1 \text {. }
$$

Brezis and Friedman [1] showed that there are singular solutions if and only if $q \in(1,1+2 / n)$. Existence of VSS for the same range $q \in(1,1+n / 2)$ was later established by Brezis, Peletier, and Terman [2]. Relations between VSS and FS were discovered by Kamin and Peletier [9]. All singular solutions of (1.7) were classified by Oswald [20]. Stability of singular solutions was studied by Galaktionov, Kurdyumov, \& Samarskii [8, and references therein]. Recently, Leoni [19] considered a similar problem for the elliptic equation $\operatorname{div}\left(|\nabla u|^{p-2} \nabla u\right)+x \cdot \nabla u^{q}+\alpha u^{q}=0$. 
In this paper, we study the case

$$
1<p<2, \quad q>1 .
$$

One notices that (1.1) becomes singular at points where $|\nabla u|=0$.

In [3], we studied self-similar singular solutions of (1.1), i.e., the ODE problem (1.5) under the constraint (1.8). We established the following result.

Proposition 1 ([3]). Assume that (1.8) holds. Then (1.5) has a non-trivial solution if and only if $q<p-1+p / n$. In addition, in the case of existence, the non-trivial solution to (1.5) is unique. In terms of (1.1), this result translates into the following:

(i) If $q<p-1+p / n$, then (1.1) has a unique self-similar VSS.

(ii) If $q \geq p-1+p / n$, then (1.1) does not have any self-similar singular solution.

Based on this result, here we classify all singular solutions of (1.1) under the assumption (1.8). In particular, we prove the following:

Theorem 1.1. Assume (1.8). Then the followings hold:

(i) every singular solution of (1.1) is either an FS or a VSS;

(ii) when $q \geq p-1+p / n$, (1.1) does not have any singular solution;

(iii) when $q<p-1+p / n$, (1.1) admits a unique VSS $u_{\infty}$ and, for every $c>0$, a unique FS $u_{c}$ with initial mass $c$. In addition, $u_{c_{1}}<u_{c_{2}}$ for any $c_{1}<c_{2}$ and $u_{c} \rightarrow u_{\infty}$ as $c \rightarrow \infty$.

The main structure of our proof of the theorem is adapted from that of Kamin and Vazquez 13 .

After the pioneering work of [1, 2, 8] on (1.7), there was work on singular solutions for the porous media equation with absorption

$$
u_{t}=\Delta u^{m}-u^{q} \quad \text { in } \mathbb{R}^{N} \times(0, \infty) .
$$

When $m>1$, i.e., the slow diffusion case, Kamin, Peletier, and Vazquez 11 provide a complete classification of singular solutions of (1.9).

(1) When $q \geq m+2 / n$, (1.9) has no singular solution at all.

(2) When $q \in(m, m+2 / n)$, there exists a unique VSS and, in addition, for every $c>0$, a unique FS with initial mass $c$. (Existence of self-similar VSS was established by Peletier and Terman [21] whereas uniqueness of VSS was established by Kamin and Veron in [14.)

(3) When $q \in(1, m]$, there does not exist any VSS but there exists, for every $c>0$, a unique FS with initial mass $c$.

The corresponding results for FS were established by Kamin and Peletier in [10].

When $m \in(\max \{0,1-2 / n\}, 1)$, i.e., the fast diffusion case, Peletier and Zhao 23 proved that (1.9) has FS and VSS if and only if $q \in(1, m+2 / n)$. Recently, Leoni 17] proved that when $m \in(0,1)$ and $q>1$, (1.9) has a self-similar VSS if and only if $m>\max \{0,1-2 / n\}$ and $q \in(1, m+2 / n)$. See also Leoni $[18$, and a recent improvement of Kwak [15, 16. More recently in [4, we obtained uniqueness of FSs and VSSs for the PDE (1.9) for $(m, q)$ in the same range as in [17.

Our paper is organized as follows. In $\S 2$, we first show that a singular solution of (1.1) is either an FS or a VSS. Then we provide two upper bounds for any singular solution of (1.1), one of which has the form $A t^{-1 /(q-1)}$ and the other has the form $B t^{1 /(2-p)}|x|^{-p /(2-p)}$. Also we show that if (1.1) has a singular solution, then (1.1) admits a maximal self-similar singular solution $u^{*}$; here maximal means that $u^{*}$ is no less than any singular solution of (1.1). As a byproduct, we conclude from 
Proposition 1 that (1.1) has no singular solution when $q \geq p-1+p / n$. For possible other applications, we also provide an alternative proof of this non-existence result which does not employ the ODE result of Proposition 1. The proof uses the fact that the integral $\int_{|x| \leq 1} \min \left\{A t^{-1 /(q-1)}, B t^{1 /(2-p)}|x|^{-p /(2-p)}\right\} d x$ converges to zero as $t \rightarrow 0$ when $q>p-1-p / n$ and is uniformly bounded in $t$ when $q=p-1+p / n$.

In $\S 3$, we establish the existence of FS and VSS when $q \in(1, p-1+p / n)$. In fact, we show that an FS with initial mass $c$ can be obtained as a limit of any sequence of solutions of (1.1), or of the more general (1.6), whose initial data approximate the measure $c \delta(\cdot)$. A VSS can be obtained as the limit, as $c \rightarrow \infty$, of FS with initial mass $c$.

At last, in $\S 4$, we prove the uniqueness of singular solutions. First we show the uniqueness of FS for the pure $p$-Laplacian evolution equation

$$
u_{t}=\operatorname{div}\left(|\nabla u|^{p-2} \nabla u\right) .
$$

The proof relies on a blow-up technique and a scaling invariance $u \rightarrow u^{h}(x, t)$ of the equation (1.1), where

$$
u^{h}:=h^{1 / k} u\left(h^{1 /(n k)} x, h t\right), \quad k:=p-2+p / n .
$$

Then we establish the uniqueness of FS for (1.1). From the existence proof in $\S 3$, one derives that an FS of (1.1) is bounded by the unique FS of (1.10) with the same initial mass, which implies that the $L^{1}\left(\mathbb{R}^{n}\right)$ difference of any two FSs of (1.1) with the same initial mass approaches zero as $t \rightarrow 0$. The uniqueness then follows from a contraction principle, which asserts that the $L^{1}\left(\mathbb{R}^{1}\right)$ difference of any two solutions of (1.1) is non-increasing in $t$.

Finally we prove the uniqueness of VSS of (1.1). We show that any VSS is an upper bound of any FS, so $u_{\infty}$, the limit of $u_{c}$ as $c \rightarrow \infty$, is the minimal VSS; i.e., it is no bigger than any other VSS. With this minimality and scaling invariance of (1.1), we show that $u_{\infty}$ is self-similar. As both $u^{*}$ and $u_{\infty}$ are self-similar VSS, by Proposition 1, they are identical, which yields the uniqueness of VSS.

\section{Properties of Singular SOlutions AND A NON-EXISTEnCE RESUlt}

In this section, we shall first establish certain properties of singular solutions of (1.1), and then prove that (1.1) does not have any singular solution when $q \geq$ $p-1+p / n$.

Lemma 2.1. Let $p>1$ and $\phi(\cdot)$ be a non-negative function on $[0, \infty)$. Assume that $u$ is a singular solution of (1.6), i.e., a non-negative, non-trivial solution of (1.6) that is continuous in $\mathbb{R}^{n} \times[0, \infty) \backslash\{(0,0)\}$ and satisfies (1.2). Then either (1.3) or (1.4) holds. In particular, taking $\phi(u)=u^{q}(q>0)$ we conclude that a singular solution of (1.1) is either an FS or a VSS.

Proof. The proof given below follows the same idea as that in [11.

By (1.2), for every $\varepsilon>0$, there exists $t_{\varepsilon}>0$ such that $\sup _{|x|>1, t \in\left(0, t_{\varepsilon}\right]} u(x, t) \leq \varepsilon$. Multiplying (1.6) by $(u-\varepsilon)_{+}^{\delta}(\delta>0)$, integrating the resulting equation over $\mathbb{R}^{n} \times(\tau, t), 0<\tau<t \leq t_{\varepsilon}$, and sending $\delta \rightarrow 0$ we then obtain

$$
\int_{\mathbb{R}^{n}}(u-\varepsilon)_{+}(x, t) d x \leq \int_{\mathbb{R}^{n}}(u-\varepsilon)_{+}(x, \tau) d x, \quad 0<\tau<t \leq t_{\varepsilon} .
$$


Thus, $\int_{\mathbb{R}^{n}}(u(\cdot, t)-\varepsilon)_{+}$is non-increasing in $t$, so that there exists $c_{\varepsilon} \in[0, \infty) \cup\{\infty\}$ such that

$$
c_{\varepsilon}=\lim _{t \searrow 0} \int_{\mathbb{R}^{n}}(u-\varepsilon)_{+}(x, t) d x=\lim _{t \searrow 0} \int_{|x|<1}(u-\varepsilon)_{+}(x, t) d x .
$$

Also noting that $c_{\varepsilon}$ is non-increasing in $\varepsilon$, sending $\varepsilon \searrow 0$ we then obtain that there exists $c \in[0, \infty) \cup\{\infty\}$ such that

$$
c=\lim _{\varepsilon \rightarrow 0} c_{\varepsilon}=\lim _{\varepsilon \searrow 0} \lim _{t \searrow 0} \int_{|x|<1}(u-\varepsilon)_{+}(x, t) d x=\lim _{t \searrow 0} \int_{|x|<1} u(x, t) d x .
$$

Notice that $c=0$ would imply, for all $\varepsilon>0$, that $c_{\varepsilon}=0$, i.e., $\sup _{x \in \mathbb{R}^{n}} u(x, t) \leq \varepsilon$ for all $t \in\left(0, t_{\varepsilon}\right)$, which, by the maximum principle, implies that $u \leq \varepsilon$ for all $(x, t) \in \mathbb{R}^{n} \times(0, \infty)$, so that, as $\varepsilon>0$ is arbitrary, $u \equiv 0$. Since $u$ is non-trivial, we must have $c \in(0, \infty) \cup\{\infty\}$. This completes the proof.

Next we provide two upper bounds for singular solutions of (1.1).

Lemma 2.2. Assume that $1<p<2$.

(i) If $q>1$ and $u$ is a singular solution $u$ of (1.1), then for $A:=\left(\frac{1}{q-1}\right)^{1 /(q-1)}$,

$$
u(x, t) \leq A t^{-1 /(q-1)} \quad \text { in } \mathbb{R}^{n} \times(0, \infty) .
$$

(ii) If $u$ is a singular solution to (1.6) with $\phi(\cdot) \geq 0$, then for

$$
\begin{gathered}
B:=\left(\frac{2(p-1) p^{p-1}}{(2-p)^{p-1}}\right)^{1 /(2-p)}, \\
u(x, t) \leq B t^{1 /(2-p)}|x|^{-p /(2-p)} \quad \text { in } \mathbb{R}^{n} \times(0, \infty) .
\end{gathered}
$$

Proof. (i) For every $\varepsilon>0$, the function $A(t-\varepsilon)^{-1 /(q-1)}$ is a solution of (1.1) in $\mathbb{R}^{n} \times(\varepsilon, \infty)$. Since $u(\cdot, \varepsilon)$ is in $L^{\infty}\left(\mathbb{R}^{n}\right)$, comparing $u$ with $A(t-\varepsilon)^{-1 /(q-1)}$ in $\mathbb{R}^{n} \times(\varepsilon, \infty)$ yields $u \leq A(t-\varepsilon)^{-1 /(q-1)}$ in $\mathbb{R}^{n} \times(\varepsilon, \infty)$. Sending $\varepsilon \searrow 0$ then gives (2.1).

(ii) Direct calculation shows that for any $\varepsilon>0$, the function $B(t+\varepsilon)^{1 /(2-p)}$ $\left(x_{1}-\varepsilon\right)^{-p /(2-p)}+\varepsilon$ is a solution to $w_{t}-\operatorname{div}\left(|\nabla w|^{p-2} \nabla w\right)=0$ in $\left\{(x, t) \mid x_{1}>\varepsilon, t \geq\right.$ $0\}$. Comparing this function with $u$ in the domain $\left\{(x, t) \mid x_{1}>\varepsilon, t \geq 0\right\}$ then gives $u(x, t) \leq B(t+\varepsilon)^{1 /(2-p)}\left(x_{1}-\varepsilon\right)^{-p /(2-p)}+\varepsilon$ for all $x_{1}>\varepsilon, t \geq 0$. Sending $\varepsilon \searrow 0$ yields $u(x, t) \leq B t^{1 /(2-p)} x_{1}^{-p /(2-p)}$ for all $x_{1}>0$ and $t \geq 0$. The assertion (2.2) then follows from the invariance of the equation for $u$ under the rotation of $x$.

Remark 2.1. If $p \in\left(\frac{2 n}{n+1}, 2\right)$, then $\frac{p}{2-p}>n$, so that, for any $R>0$ and any singular solution $u$ of (1.6), $\int_{|x|>R} u(x, t) d x \leq B t^{1 /(2-p)} \int_{|x|>R}|x|^{-p /(2-p)} d x \rightarrow 0$ as $t \searrow 0$. Thus, (1.3) and (1.4) are equivalent to $\lim _{t \searrow 0} \int_{\mathbb{R}^{n}} u(x, t) d x=c$ and $\lim _{t \searrow 0} \int_{\mathbb{R}^{n}} u(x, t) d x=\infty$, respectively.

With the upper bounds for singular solutions, we can now show that if (1.1) has a singular solution, then there exists a maximal singular solution, which has to be self-similar. 
Lemma 2.3. Let $q>1$ and $p \in(1,2)$. Also assume that (1.1) has a singular solution. Then (1.1) admits a singular solution $u^{*}$ having the following properties:

(1) Every singular solution of (1.1) is no bigger than $u^{*}$; namely, $u^{*}$ is the maximal singular solution of (1.1).

(2) $u^{*}$ is self-similar; namely, there exists a smooth function $w(\cdot):[0, \infty) \rightarrow$ $[0, \infty)$ such that $u^{*}=t^{1 /(q-1)} w\left(|x| t^{-(q+1-p) /[(q-1) p]}\right)$ and $w$ solves (1.5).

Proof. For each $\tau>0$, let $u_{\tau}(x, t)$ be the solution of (1.1) in $\mathbb{R}^{n} \times(\tau, \infty)$ with initial value

$$
u_{\tau}(x, \tau)=\min \left\{A \tau^{-1 /(q-1)}, B \tau^{1 /(2-p)}|x|^{-p /(2-p)}\right\} \quad \text { on } \quad \mathbb{R}^{n} \times\{t=\tau\} .
$$

Then as in the proof of the previous lemma,

$$
u_{\tau}(x, t) \leq \min \left\{A t^{-1 /(q-1)}, B t^{1 /(2-p)}|x|^{-p /(2-p)}\right\} \quad \forall(x, t) \in \mathbb{R}^{n} \times[\tau, \infty) .
$$

Consequently, for any $\tau_{1}>\tau_{2}>0, u_{\tau_{1}}\left(\cdot, \tau_{1}\right) \geq u_{\tau_{2}}\left(\cdot, \tau_{1}\right)$ so that by comparison, $u_{\tau_{1}} \geq u_{\tau_{2}}$ in $\mathbb{R}^{n} \times\left[\tau_{1}, \infty\right)$. Hence, $\lim _{\tau \backslash 0} u_{\tau}$ exists for all $(x, t) \in \mathbb{R}^{n} \times(0, \infty)$. We denote this limit by $u^{*}$, which is necessarily a solution of (1.1). Since each $u_{\tau}$ satisfies (2.3), $u^{*}(x, t) \leq\left\{A t^{-1 /(q-1)}, B t^{1 /(2-p)}|x|^{-p /(2-p)}\right\}$. It then follows that $u^{*}$ satisfies (1.2).

To show that $u^{*}$ is non-trivial, we need only show that $u^{*}$ is no less than any singular solution of (1.1). In fact, if $u$ is a singular solution of (1.1), then from Lemma 2.2 and a comparison principle, $u \leq u_{\tau}$ in $\mathbb{R}^{n} \times[s, \infty)$ for any $0<\tau \leq s$. Thus, $u \leq u^{*}$ in $\mathbb{R}^{n} \times(s, \infty)$ for any $s>0$; i.e., $u \leq u^{*}$ in $\mathbb{R}^{n} \times(0, \infty)$. Thus, $u^{*}$ is non-trivial and is the maximal singular solution of (1.1) if (1.1) has a singular solution.

It remains to show that $u^{*}$ is self-similar. From the construction of $u^{*}$, we see that $u^{*}$ is radially symmetric. Note that for any $\lambda>0$, the function $\mathcal{T}^{\lambda}\left(u^{*}\right):=$ $\lambda^{1 /(q-1)} u^{*}\left(\lambda^{\frac{q+1-p}{p(q-1)}} x, \lambda t\right)$ is a non-trivial and non-negative solution of (1.1) satisfying (1.2), so it is a singular solution of (1.1). Since $u^{*}$ is maximal, $u^{*} \geq \mathcal{T}^{\lambda}\left(u^{*}\right)$ for all $\lambda>0$. Observe that the operator $\mathcal{T}^{\lambda}$ preserves the order; namely, if $u_{1} \leq u_{2}$, then $\mathcal{T}^{\lambda}\left(u_{1}\right) \leq \mathcal{T}^{\lambda}\left(u_{2}\right)$ for all $\lambda>0$. We then obtain from $u^{*} \geq \mathcal{T}^{\lambda}\left(u^{*}\right)$ that $\mathcal{T}^{\ell}\left(u^{*}\right) \geq \mathcal{T}^{\ell}\left(\mathcal{T}^{\lambda}\left(u^{*}\right)\right)$ for all $\ell>0$ and $\lambda>0$. In particular taking $\lambda=1 / \ell$ and using $\mathcal{T}^{\ell}\left(\mathcal{T}^{1 / \ell}\left(u^{*}\right)\right)=u^{*}$ we get $\mathcal{T}^{\ell}\left(u^{*}\right) \geq u^{*}$. Hence, $u^{*}=\mathcal{T}^{\ell}\left(u^{*}\right)$ for all $\ell>0$. Thus, $u$ is self-similar and can be written in the form $t^{1 /(q-1)} w\left(|x| t^{-\frac{q+1-p}{(q-1) p}}\right)$ for some $w$. This completes the proof.

Now we are ready to prove the non-existence of singular solutions of (1.1) when $q \geq p-1+p / n$.

Theorem 2.4. Assume $p$ and $q$ satisfy (1.8) and $q \geq p-1+p / n$. Then (1.1) does not have any singular solution.

Proof. According to our ODE result in Proposition 1, problem (1.1) does not have any self-similar singular solution when (1.8) and $q \geq p-1+p / n$ hold. The assertion of the theorem then follows from Lemma 2.3 .

The above proof relies on the analysis of the ODE problem (1.5), i.e., Proposition 1. Below we provide another proof, which does not use any of the ODE result. The method may be applied to some other similar problems. 
Another proof of Theorem 2.4. Suppose for the contrary that (1.1) has a singular solution $u$. Then for any $t>0$ and $R \in[0,1$ ), applying Lemma 2.2 (i) for $|x|<R$ and (ii) for $|x|>R$ yields

$$
\int_{|x| \leq 1} u(x, t) d x \leq \int_{|x| \leq R} A t^{-1 /(q-1)} d x+\int_{R<|x|<1} B t^{1 /(2-p)}|x|^{-p /(2-p)} d x
$$

We consider several different cases.

Case (1): $p<\frac{2 n}{n+1}$. In this case, $\frac{p}{2-p}<n$ so that taking $R=0$ in (2.4) and denoting by $\omega_{n}$ the area of unit sphere in $\mathbb{R}^{n}$, we obtain

$$
\int_{|x| \leq 1} u(x, t) d x \leq B t^{1 /(2-p)}\left(n-\frac{p}{2-p}\right)^{-1} \omega_{n} \rightarrow 0 \quad \text { as } t \searrow 0 .
$$

But this contradicts Lemma 2.1. which asserts that $u$ satisfies either (1.3) or (1.4). Hence, there are no singular solutions when $p<\frac{2 n}{n+1}$.

Case (2): $p=\frac{2 n}{n+1}$ and $q>1$. In this case $\frac{p}{2-p}=n$, so taking

$$
R=t^{[1 /(q-1)+1 /(2-p)] / n}
$$

in (2.4) we obtain

$$
\int_{|x| \leq 1} u(x, t) d t \leq t^{1 /(2-p)} \omega_{n}\{A / n+B[1 /(q-1)+1 /(2-p)] / n|\ln t|\} \rightarrow 0 \text { as } t \searrow 0 .
$$

Hence, as in case (i) we get a contradiction.

Case (3): $p>\frac{2 n}{n+1}$ and $q>p-1+p / n$. In this case $\frac{p}{2-p}>n$, so taking $R=t^{(q+1-p) /[p(q-1)]}$ in (2.4) gives

$$
\begin{gathered}
\int_{|x| \leq 1} u(x, t) d x \leq A \omega_{n} n^{-1} t^{-1 /(q-1)} R^{n}+B \omega_{n}\left(\frac{p}{2-p}-n\right)^{-1} t^{1 /(2-p)} R^{n-p /(2-p)} \\
=\left\{A n^{-1}+B\left(\frac{p}{2-p}-n\right)^{-1}\right\} \omega_{n} t^{n[q-(p-1+p / n)] /[p(q-1)]} \rightarrow 0 \quad \text { as } t \searrow 0 .
\end{gathered}
$$

Again, this is impossible.

Case (4): $p>\frac{2 n}{n+1}$ and $q=p-1+p / n$. In this case, we still have $\frac{p}{2-p}>n$, so from (2.2), $u(\cdot, t) \in L^{1}\left(\mathbb{R}^{n}\right)$ for all $t>0$. In addition, as $q>1$ and $u(\cdot, t)$ is uniformly bounded for every fixed $t>0, u^{q}(\cdot, t) \in L^{1}\left(\mathbb{R}^{n}\right)$. Hence, integrating (1.1) over $\mathbb{R}^{n}$ yields that

$$
\frac{d}{d t} \int_{\mathbb{R}^{n}} u(x, t) d x=-\int_{\mathbb{R}^{n}} u^{q}(x, t) d x \quad \forall t>0 .
$$

Define $e(t)=\int_{\mathbb{R}^{n}} u(x, t) d t$ and denote by $R=R(t)$ the positive constant such that

$$
2 B \omega_{n}\left(\frac{p}{2-p}-n\right)^{-1} t^{1 /(2-p)} R^{n-p /(2-p)}=e(t) .
$$

Then, by the estimate (2.2),

$$
\begin{aligned}
\int_{|x| \leq R} u(x, t) d x & =\int_{\mathbb{R}^{n}} u(x, t) d x-\int_{|x|>R} u(x, t) d x \\
& \geq e(t)-\int_{|x| \geq R} B t^{1 /(2-p)}|x|^{-p /(2-p)} d x \\
& =e(t)-B t^{1 /(2-p)} \omega_{n}\left(\frac{p}{2-p}-n\right)^{-1} R^{n-p /(2-p)}=\frac{1}{2} e(t)
\end{aligned}
$$


by the definition of $R(t)$. Consequently, by Cauchy's inequality,

$$
\frac{1}{2} e(t) \leq \int_{|x|<R} u(x, t) d x \leq\left(\frac{\omega_{n}}{n} R^{n}\right)^{1-1 / q}\left(\int_{|x|<R} u^{q} d x\right)^{1 / q},
$$

so that

$$
\int_{\mathbb{R}^{n}} u^{q} \geq \int_{|x|<R} u^{q} \geq\left(\frac{1}{2} e(t)\right)^{q}\left(\frac{\omega_{n}}{n} R^{n}\right)^{1-q}=B_{1}(e(t))^{1+p / n} t^{-1}
$$

by the definition of $R(t)$ and the equality $q=p-1+p / n$, where $B_{1}$ is a positive constant depending only on $p, q$, and $n$. It then follows from (2.5) that $\frac{d}{d t} e(t) \leq$ $-B_{1} e^{1+p / n}(t) t^{-1}$ for all $t>0$. Integrating this inequality over $(\tau, 1]$ then yields that

$$
\frac{p}{n}\left\{e^{-p / n}(1)-e^{-p / n}(\tau)\right\} \geq-B_{1} \ln \tau \quad \forall \tau \in(0,1) .
$$

Sending $\tau \searrow 0$ we get a contradiction.

Summarizing Cases (1)-(4), we conclude that (1.1) has no singular solution when $q \geq p-1+p / n$ and $p \in(1,2)$.

Remark 2.2. In case (1) of the proof, we only used Lemma 2.2 (ii) and Lemma 2.1. so when $p \in\left(1, \frac{2 n}{n+1}\right)$, problem (1.6) has no singular solution, provided that $\phi$ is non-negative. In particular, (1.10) has no singular solution when $1<p<\frac{2 n}{n+1}$.

Remark 2.3. In proving the same non-existence theorem for the porous media equation $u_{t}=\Delta u^{m}-u^{q}$, where $q>m+2 / n$, in [10] (for $m>1$ ) and in [23] (for $m \in(\max \{1-2 / n, 0\}, 1))$ the integral identity $\int_{\mathbb{R}^{n}} \zeta \Delta u^{m}=\int u^{m} \Delta \zeta$ for a smooth test function $\zeta$ plays an essential role. Due to the quasi-linear nature of the $p$ Laplacian operator, this technique cannot be used here. The proof provided here is elementary and straightforward and can be applied to the porous media equation with absorption to show the non-existence of singular solutions. It can also be applied to the non-existence of a singular solution to (1.6), where $\phi(\cdot)$ is continuous, non-negative and satisfies $\liminf \operatorname{in}_{u \rightarrow \infty} \phi(u) u^{-q}>0$ for $q=p-1+p / n$.

\section{EXISTENCE OF SINGULAR SOLUTIONS}

In the rest of this paper, we always assume that

$$
1<q<p-1+p / n, \quad p<2 .
$$

We remark that the first condition implies that $1<p-1+p / n$, i.e., $p>\frac{2 n}{n+1}$.

In this section, we shall establish the existence of FS and VSS for (1.1) under the assumption (3.1). For this purpose, we cite a few known results from [6] concerning the parabolic $p$-Laplacian equation for $p \in\left(\frac{2 n}{n+1}, 2\right)$.

Existence [6]: Assume that $\phi(\cdot)$ is non-negative and continuous. Then for any bounded and non-negative initial data, (1.6) has a unique (weak) solution.

Regularity [6]: Assume that $u$ is a non-negative locally bounded solution of (1.6). Then both $u$ and $\nabla u$ are locally Hölder continuous with the Hölder exponent and the Hölder norm depending only on $p, n$, and the local $L^{\infty}$ bound of the solution. 
$L^{\infty}$ bound [6. p. 127, Corollary 5.1]: Assume that $u$ is a non-negative solution of (1.10). Then there exists a positive constant $M=M(p, n)$ such that for every $(x, t) \in \mathbb{R}^{n} \times(0, \infty)$,

$$
u(x, t) \leq M t^{-1 /(p-2+p / n)}\left(\frac{1}{t} \int_{0}^{t} \int_{|y-x|<1} u(y, \tau) d y d \tau\right)^{p /[n p-2 n+p]}+M t^{1 /(2-p)}
$$

Contraction Principle: Assume that $\phi(\cdot)$ is non-decreasing and $u_{1}$ and $u_{2}$ are solutions of $(1.6)$ on $\Omega \times(0, \infty), \Omega \subseteq \mathbb{R}^{n}$, such that $u_{1} \leq u_{2}$ near the lateral boundary $\partial \Omega \times(0, \infty)$. Then the function $t \rightarrow \int_{\Omega}\left(u_{1}(x, t)-u_{2}(x, t)\right)+d x$ is nonincreasing. In particular, if $\Omega=\mathbb{R}^{n}$, then the function $t \rightarrow \int_{\mathbb{R}^{n}}\left|u_{1}(x, t)-u_{2}(x, t)\right| d x$, if bounded, is non-increasing in $t$.

The contraction principle follows by multiplying (1.6) by $\left(u_{1}-u_{2}\right)_{+}^{\delta}(\delta>0)$, integrating over $\mathbb{R}^{n}$, and then sending $\delta \rightarrow 0$.

We can now establish the existence of FS for (1.1). In fact, we do it for (1.6).

Theorem 3.1. Assume that $\phi(\cdot):[0, \infty) \rightarrow[0, \infty)$ is a continuous and nondecreasing function satisfying, for some positive constant $C$,

$$
0 \leq \phi(u) \leq C u\left(1+u^{q-1}\right) \quad \forall u \geq 0 .
$$

Let $c>0$ be given and let $\left\{\varphi_{j}(\cdot)\right\}_{j=1}^{\infty}$ be a c $\delta$-sequence; namely, $\varphi_{j}$ is continuous, non-negative,

$$
\int_{\mathbb{R}^{n}} \varphi_{j} d x=c \quad \text { and } \quad \lim _{j \rightarrow \infty} \int_{|x| \geq r} \varphi_{j}(x) d x=0 \quad \forall r>0 .
$$

Let $u_{j}$ be the solution to (1.6) with initial data $u_{j}(\cdot, 0)=\varphi_{j}$.

Then $\lim _{j \rightarrow \infty} u_{j}$ exists and is a fundamental solution of (1.6) with initial mass c.

Proof. Let $u_{j}^{o}$ be the corresponding solution with $\phi \equiv 0$. Since $\left\|u_{j}^{o}(\cdot, t)\right\|_{L^{1}\left(\mathbb{R}^{n}\right)}=c$ for all $t \geq 0$, it follows from (3.2) that

$$
0 \leq u_{j}^{o}(x, t) \leq M(p, n, c)\left\{t^{-1 /[p-2+p / n]}+t^{1 /(2-p)}\right\} \quad \forall t>0 .
$$

As $u_{j} \leq u_{j}^{o},\left\{u_{j}\right\}$ is locally uniformly bounded. Consequently, by the regularity result [6] for locally bounded solutions of (1.6), the family $\left\{u_{j}\right\}_{j=1}^{\infty}$ is equicontinuous in any compact subset of $\mathbb{R}^{n} \times(0, \infty)$. Hence, we can find a function $u$ and a subsequence, which we still denote by $\left\{u_{j}\right\}$, such that, as $j \rightarrow \infty, u_{j} \rightarrow u$ uniformly in any compact subset of $\mathbb{R}^{n} \times(0, \infty)$. The limit function $u$ is necessarily a (weak) solution of (1.6) in $\mathbb{R}^{n} \times(0, \infty)$.

Now we show that $u$ is a fundamental solution of (1.6) with initial mass $c$. First of all, by Fatou's lemma,

$$
\int_{\mathbb{R}^{n}} u(x, t) d x \leq \liminf _{j \rightarrow \infty} \int_{\mathbb{R}^{n}} u_{j}(x, t) d x \leq c \quad \forall t>0 .
$$

Next, we show that for any $\delta>0$,

$$
\lim _{t \searrow 0} \int_{|x|<\delta} u(x, t) d x=c .
$$

For this purpose, let $\left\{\tilde{\varphi}_{j}(x)\right\}$ be a sequence such that $\tilde{\varphi}_{j}$ is continuous and compactly supported in $\left\{x|| x \mid \leq \delta_{j}\right\}$ with $\lim _{j \rightarrow \infty} \delta_{j} \rightarrow 0$, that $\tilde{\varphi}_{j} \leq \varphi_{j}$, and that $\int_{|x|<\delta_{j}} \tilde{\varphi}_{j} \rightarrow c$ as $j \rightarrow \infty$. Since $\left\{\varphi_{j}\right\}$ is a $c \delta$-sequence, such $\left\{\tilde{\varphi}_{j}\right\}$ exists. 
Now let $\tilde{u}_{j}$ be the solution of (1.6) with initial data $\tilde{\varphi}_{j}$. Notice that the function $B t^{1 /(2-p)}\left(x_{1}-\delta_{j}\right)^{-p /(2-p)}$ is a solution to $u_{t}=\operatorname{div}\left(|\nabla u|^{p-2} \nabla u\right)$ in $\{(x, t) \mid t \geq$ $\left.0, x_{1}>\delta_{j}\right\}$. Comparing this function with $\tilde{u}_{j}$ in $\left\{(x, t) \mid t \geq 0, x_{1} \geq \delta_{j}\right\}$ then yields that $\tilde{u}_{j} \leq B t^{1 /(2-p)}\left(x_{1}-\delta_{j}\right)^{-p /(2-p)}$. By rotational invariance, we then obtain

$$
\tilde{u}_{j}(x, t) \leq B t^{1 /(2-p)}\left(|x|-\delta_{j}\right)^{-p /(2-p)} \quad \forall t \geq 0 \text { and }|x| \geq \delta_{j} .
$$

Now we estimate the total mass of $\tilde{u}_{j}(\cdot, t)$. From the differential equation, we have

$$
\int_{\mathbb{R}^{n}} \tilde{u}_{j}(x, t) d x=\int_{\mathbb{R}^{n}} \tilde{\varphi}_{j}(x) d x-\int_{0}^{t} \int_{\mathbb{R}^{n}} \phi\left(\tilde{u}_{j}\right) d x d \tau .
$$

As $u_{j}^{o} \geq u_{j} \geq \tilde{u}_{j}$, we can use the $L^{\infty}$ bound of $u_{j}^{o}$ in (3.3) and the assumption that $0 \leq \phi(s) \leq C s\left[1+s^{q-1}\right]$ to obtain

$$
\begin{aligned}
\int_{0}^{t} \int_{\mathbb{R}^{n}} \phi\left(\tilde{u}_{j}\right) & \leq\left(\sup _{\tau \in(0, t)} \int_{\mathbb{R}^{n}} \tilde{u}_{j}(x, \tau) d x\right)\left(\int_{0}^{t} \sup _{x \in \mathbb{R}^{n}} C\left[1+\left(u_{j}^{o}\right)^{q-1}\right] d t\right) \\
& \leq c \int_{0}^{t} C\left[1+M t^{-(q-1) /[p-2+p / n]}+M t^{(q-1) /(2-p)}\right] \\
& \leq \hat{M}(p, n, c, q) t^{\frac{(p-1+n / p)-q}{p-2+p / n}} \quad \forall t \in(0,1] .
\end{aligned}
$$

Thus, for any fixed $t>0$ and large $j$ such that $\delta>\delta_{j}$,

$$
\begin{aligned}
\int_{|x| \leq \delta} u_{j}(x, t) d x \geq & \int_{|x| \leq \delta} \tilde{u}_{j} d x \\
\geq & \int_{\mathbb{R}^{n}} \tilde{u}_{j}(x, t) d x-\int_{|x|>\delta} B t^{p /(2-p)}\left|x-\delta_{j}\right|^{-p /(2-p)} d x \\
\geq & \int_{\mathbb{R}^{n}} \tilde{\varphi}_{j}(x) d x-\hat{M} t^{\frac{(p-1+p / n)-q}{p-2+p / n}} \\
& -B t^{p /(2-p)} \int_{|x| \geq \delta}\left(|x|-\delta_{j}\right)^{-\frac{p}{2-p}} d x .
\end{aligned}
$$

As $p /(2-p)>n$ and $q<p-1+p / n$, sending $j \rightarrow \infty$ we then obtain

$$
\int_{|x|<\delta} u(x, t) d x \geq c-M(p, q, n, c)\left\{t^{\frac{(p-1-p / n)-q}{p-2+p / n}}+t^{\frac{p}{2-p}}\right\} .
$$

Sending $t \searrow 0$ we then obtain (3.4).

Finally, we show that $u$ satisfies (1.2). Using the contraction principle (since $\phi$ is non-increasing), we have, for any $t>0$,

$$
\begin{aligned}
& \int_{x_{1}>\varepsilon}\left(u_{j}^{o}(x, t)-B(t+\varepsilon)^{1 /(2-p)}\left(x_{1}-\varepsilon\right)^{-p /(2-p)}\right)_{+} d x \\
\leq & \int_{x_{1}>\varepsilon}\left(u_{j}^{o}(x, 0)-B \varepsilon^{1 /(2-p)}\left(x_{1}-\varepsilon\right)^{-p /(2-p)}\right)_{+} d x .
\end{aligned}
$$

As $u_{j}^{o}(\cdot, 0)=\varphi_{j}$ and $\int_{|x| \geq \varepsilon} \varphi_{j}(x) d x \rightarrow 0$ as $j \rightarrow \infty$, sending $j \rightarrow \infty$ and using $u \leq \lim \sup u_{j}^{o}$, we obtain $\left(u(x, t)-B(t+\varepsilon)^{2-p}\left(x_{1}-\varepsilon\right)^{-p /(2-p)}\right)_{+}=0$ in $\left\{x \mid x_{1}>\varepsilon\right\}$, so that $u(x, t) \leq B(t+\varepsilon)^{1 /(2-p)}\left(x_{1}-\varepsilon\right)$ if $x_{1}>\varepsilon$. Sending $\varepsilon \rightarrow 0$ and using the rotational invariance, we then obtain the estimate

$$
u(x, t) \leq B t^{1 /(2-p)}|x|^{-p /(2-p)} .
$$

This shows that $u$ satisfies (1.2). Hence, $u$ is an FS with initial mass $c$. 
As we shall show later, FSs are unique, so the whole sequence $\left\{u_{j}\right\}$ converges to $u$.

The following two corollaries follow by taking $\phi \equiv 0$ and $\phi=u^{q}$ in Theorem 3.1.

Corollary 3.2. Assume $p \in\left(\frac{2 n}{n+1}, 2\right)$. Then for every $c>0$, (1.10) has an FS with initial mass $c$.

Corollary 3.3. Assume (3.1). Then for any $c>0$, (1.1) has an FS with initial mass $c$.

Next we establish the existence of VSS of (1.1).

Theorem 3.4. Assume (3.1). Then (1.1) has a VSS $u_{\infty}$ which is the limit, as $c \rightarrow \infty$, of the FS of (1.1) with initial mass $c$.

Proof. Let $\zeta(\cdot)$ be any non-negative continuous function on $\mathbb{R}^{n}$ satisfying $\int_{\mathbb{R}^{n}} \zeta(x) d x$ $=1$. Define $\varphi_{j}^{c}=c j^{-n} \zeta\left(\frac{x}{j}\right)$ for all real $c>0$ and all integers $j \geq 1$. Then $\left\{\varphi_{j}^{c}\right\}_{j=1}^{\infty}$ is a $c \delta$-sequence, and we can apply Theorem 3.1 to obtain an FS $u_{c}$ of (1.1). Since for each $j, \varphi_{j}^{c}$ is monotonic in $c, u_{c}$ is monotonic in $c$. Consequently, $u_{\infty}(x, t)=\lim _{c \rightarrow \infty} u_{c}(x, t)$ exists. By the uniform estimates (2.1) and (2.2) for FSs, we know that $u_{\infty}(\cdot, t)$ is bounded for each $t>0$ and satisfies also the estimates (2.1) and (2.2). By the local equicontinuity of $\left\{u_{c}\right\}$ (since they are locally uniformly bounded), $u_{\infty}$ is a (weak) solution of (1.1). Also, the estimate (2.2) for $u=u_{\infty}$ shows that $u_{\infty}$ satisfies (1.2). Finally, since $u \geq u_{c}$ for every $c$,

$$
\liminf _{t \searrow 0} \int_{|x|<1} u_{\infty}(x, t) d x \geq \lim _{t \searrow 0} \int_{|x|<1} u_{c}(x, t) d x=c \quad \forall c>0 .
$$

Thus, $u_{\infty}$ satisfies (1.3). That is, $u_{\infty}$ is a VSS of (1.1).

Remark 3.1. The same proof can be applied to show that (1.6) admits a VSS when $\phi$ is continuous, non-negative and non-decreasing, and satisfies $\int^{\infty} \frac{1}{\phi(s)} d s<\infty$ and $\phi(s) \leq C s\left(1+s^{q-1}\right)$ for some $q<p-1+p / n$.

\section{UNIQUENESS OF SINGULAR SOLUTIONS}

In this section, we prove the uniqueness of singular solutions of (1.1) under (3.1). To do this, we first show the uniqueness of FS for (1.10), then the uniqueness of FS for (1.1), and finally the uniqueness of VSS for (1.1).

\subsection{Uniqueness of FS for $u_{t}=\operatorname{div}\left(|\nabla u|^{p-2} \nabla u\right)$.}

Theorem 4.1. Assume that $p \in\left(\frac{2 n}{n+1}, 2\right)$. Then for every $c>0$, (1.10) has a unique $F S E_{c}$ with initial mass $c$. It is given by

$$
E_{c}(x, t)=G t^{-1 / k}\left\{D(c)+\left(|x| t^{-1 /(n k)}\right)^{p /(p-1)}\right\}^{-(p-1) /(2-p)}
$$

where

$$
k=k(p, n):=p-2+p / n, \quad G=G(p, n):=\left(\frac{p}{2-p}\right)^{(p-1) /(2-p)}(n k)^{1 /(2-p)},
$$

and $D(c)$ is the unique constant such that $\int_{\mathbb{R}^{n}} G\left[D(c)+|x|^{p /(p-1)}\right]^{-(p-1) /(2-p)} d x=$ $c$. 
Proof. Notice that $E_{c}$ is invariant under the scaling

$$
E(x, t) \rightarrow E^{h}:=h^{1 / k} E\left(h^{1 /(n k)} x, h t\right) \forall h>0
$$

so that

$$
\int_{\mathbb{R}^{n}} E_{c}(x, t) d x=\int_{\mathbb{R}^{n}} E_{c}(x, 1) d x=c \forall t>0 .
$$

In addition, it is easy to verify that $\lim _{t \backslash 0} E_{c}(x, t)=0$ for all $x \neq 0$, and $\int_{|x|<1} E_{c}(x, t) d x=\int_{|y|<t^{-\frac{1}{n k}}} E_{c}(y, 1) d y \rightarrow c$ as $t \searrow 0$. Direct differentiation shows that $E_{c}$ satisfies (1.10). Hence, $E_{c}$ is an FS of (1.10) with initial mass $c$.

Next we prove the uniqueness. Assume that $u$ is any fundamental solution of (1.10) with initial mass $c$. We want to show that $u=E_{c}$. We divide the proof into three steps.

Step 1. Consider the family $\left\{u^{h}\right\}_{h>0}$, where $u^{h}(x, t)=h^{1 / k} u\left(h^{1 /(n k)} x, h t\right)$.

Direct calculation shows that each $u^{h}$ is a solution of (1.10). In addition, from Remark 2.1

$$
\int_{\mathbb{R}^{n}} u^{h}(x, t) d x=\int_{\mathbb{R}^{n}} u(y, h t) d y=c \quad \forall h>0, \quad t>0 .
$$

Furthermore, using Lemma 2.1(ii),

$$
u^{h}(x, t) \leq B t^{1 /(2-p)}|x|^{-p /(2-p)} .
$$

Hence, by the regularity of solutions of (1.10), the family $\left\{u^{h}(\cdot, 1)\right\}_{h>0}$ is equicontinuous in any bounded domain of $\mathbb{R}^{n}$, so that there exists a sequence $\left\{h_{j}\right\}_{j=1}^{\infty}$ satisfying $h_{j} \searrow 0$ as $j \rightarrow \infty$ and a function $u^{o}$ such that $u^{h_{j}}(\cdot, 1) \rightarrow u^{o}(\cdot)$ uniformly in any compact subset of $\mathbb{R}^{n}$. As $p /(2-p)>n$ and $u^{h}(\cdot, 1) \leq B|x|^{-p /(2-p)}$, the Lebesgue dominated convergence theorem then gives that

$$
u^{h_{j}}(\cdot, 1) \longrightarrow u^{o} \quad \text { in } L^{1}\left(\mathbb{R}^{n}\right) .
$$

Let $v(x, t)$ be the solution to (1.10) in $\mathbb{R}^{n} \times(1, \infty)$ with initial data $v(\cdot, 1)=u^{o}$. Then, as both $u^{h_{j}}$ and $v$ are solutions of (1.10), the contraction principle shows that, for all $t \geq 1$,

$$
\int_{\mathbb{R}^{n}}\left|u^{h_{j}}(\cdot, t)-v(\cdot, t)\right| \leq \int_{\mathbb{R}^{n}}\left|u^{h_{j}}(\cdot, 1)-v(\cdot, 1)\right| \rightarrow 0 \text { as } j \rightarrow \infty .
$$

Step 2. Denote, for each $h>0$,

$$
e^{h}(t)=\int_{\mathbb{R}^{n}}\left|u^{h}(\cdot, t)-E_{c}(\cdot, t)\right|
$$

By the contraction principle, $e^{h}(t)$ is a non-increasing function of $t$. Also, for any $h>0$, by the scaling invariance $E_{c}=E_{c}^{h}$,

$$
\begin{aligned}
e^{h}(t) & =\int_{\mathbb{R}^{n}}\left|u^{h}(\cdot, t)-E_{c}^{h}(\cdot, t)\right|=h^{1 / k} \int_{\mathbb{R}^{n}}\left|u\left(h^{1 /(n k)} x, h t\right)-E\left(h^{1 /(n k)} x, h t\right)\right| d x \\
& =\int_{\mathbb{R}^{n}}\left|u(y, h t)-E_{c}(y, h t)\right| d y=e^{1}(h t) .
\end{aligned}
$$

Thus $e^{h}(t)$ is non-increasing in both $t$ and $h$. Since the initial mass of $u$ and $E_{c}$ is $c, e^{h}(t)$ is bounded by $2 c$ for all $h$ and $t$. It then follows that $\lim _{h \searrow_{0}} e^{h}(t)$ exists, and

$$
\lim _{h \searrow 0} e^{h}(1)=\lim _{h \searrow 0} e^{1}(h)=\lim _{h \searrow 0} e^{1}(2 h)=\lim _{h \searrow 0} e^{h}(2) .
$$


Denote the limit by $e^{o}$. Then, in view of (4.1) and (4.2) we obtain

$$
\begin{aligned}
e^{o} & =\lim _{j \rightarrow \infty} e^{h_{j}}(1)=\lim _{j \rightarrow \infty} \int_{\mathbb{R}^{n}}\left|u^{h_{j}}(\cdot, 1)-E_{c}(\cdot, 1)\right|=\int_{\mathbb{R}^{n}}\left|v(\cdot, 1)-E_{c}(\cdot, 1)\right| \\
& =\lim _{j \rightarrow \infty} e^{h_{j}}(2)=\int_{\mathbb{R}^{n}}\left|v(\cdot, 2)-E_{c}(\cdot 2)\right| .
\end{aligned}
$$

Step 3. We first show that $e^{o}=0$. Suppose to the contrary that $e^{o}>0$. We define $\bar{u}$ and $\underline{u}$ as the solutions of $(1.10)$ in $\mathbb{R}^{n} \times(1, \infty)$ with initial data

$$
\bar{u}(\cdot, 1):=\max \left\{v(\cdot, 1), E_{c}(\cdot, 1)\right\}, \quad \underline{u}(\cdot, 1):=\min \left\{v(\cdot, 1), E_{c}(\cdot, t)\right\} .
$$

The comparison principle then gives

$$
\bar{u} \geq \max \left\{v, E_{c}\right\} \geq \min \left\{v, E_{c}\right\} \geq \underline{u} \quad \text { in } \mathbb{R}^{n} \times[1, \infty) .
$$

Since $v(\cdot, 2) \not \equiv E_{c}(\cdot, 2)$ and $\int_{\mathbb{R}^{n}} E_{c}(\cdot, 2)=\int_{\mathbb{R}^{n}} v(\cdot, 2)=c$, there are points $x$ where $v(x, 2)=E_{c}(x, 2)$. Thus, as $\bar{u}(\cdot, 2)>\underline{u}(\cdot, 2)$,

$$
\begin{aligned}
\int_{\mathbb{R}^{n}}[\bar{u}(\cdot, 2)-\underline{u}(\cdot, 2)] & >\int_{\mathbb{R}^{n}}\left[\max \left\{v(\cdot, 2), E_{c}(\cdot, 2)\right\}-\min \left\{v(\cdot, 2), E_{c}(\cdot, 2)\right\}\right] \\
& =\int_{\mathbb{R}^{n}}\left|v(\cdot, 2)-E_{c}(\cdot, 2)\right|=e^{o}
\end{aligned}
$$

On the other hand, by the contraction principle,

$$
\int_{\mathbb{R}^{n}}|\bar{u}(\cdot, 2)-\underline{u}(\cdot, 2)| \leq \int_{\mathbb{R}^{n}}|\bar{u}(\cdot, 1)-\underline{u}(\cdot, 1)|=\int_{\mathbb{R}^{n}}\left|v(\cdot, 1)-E_{c}(\cdot, 1)\right|=e^{o} .
$$

Hence we obtain a contradiction. This contradiction shows that $e^{o}=0$.

As $e^{1}(t)$ is non-increasing in $t, 0=e^{o}=\lim _{t \backslash 0} e^{1}(t)$ then implies that $e^{1}(t)=0$ for all $t>0$. Thus, $u \equiv E_{c}$. The proof is completed.

\subsection{Uniqueness of FS of $u_{t}=\operatorname{div}\left(|\nabla u|^{p-2} \nabla u\right)-u^{q}$.}

Theorem 4.2. Assume (3.1). Then for any given $c>0$, (1.1) admits a unique fundamental solution $u_{c}$ with initial mass $c$. In addition, $u_{c}$ is monotonic in $c$.

Proof. We need only prove the uniqueness of FS. The following proof follows the idea of [13].

Let $v$ be an FS of (1.1) with initial mass $c$. We first show that $v \leq E_{c}$. In fact, for every $\tau>0$, let $v_{\tau}$ be the solution to (1.10) for $t>\tau$ with initial value $v_{\tau}=v$ on $\{t=\tau\}$. Then by comparison, $v_{\tau} \geq v$ for all $t>\tau$, so that when $\tau_{1} \leq \tau_{2}, v_{\tau_{1}} \geq v_{\tau_{2}}$ for all $t>\tau_{2}$, i.e., $\left\{v_{\tau}\right\}_{\tau>0}$ is monotonic decreasing in $\tau$. Consequently, the limit function $w=\lim _{\tau \backslash 0} v_{\tau}$ exists there.

By the upper bound for singular solutions (Lemma 2.2) and local regularity of solutions of (1.6), we know that for any $t>0, v_{\tau}(\cdot, t) \rightarrow w$, as $\tau \searrow 0$, uniformly in any compact set of $\mathbb{R}^{n}$ and in $L^{1}\left(\mathbb{R}^{n}\right)$. As $\int_{\mathbb{R}^{n}} v_{\tau}(x, t) d x$ is a constant equal 
to $\int_{\mathbb{R}^{n}} v(x, \tau) d x$, which, by Remark 2.1, approaches $c$ as $\tau \rightarrow 0$, we conclude that $\int_{\mathbb{R}^{n}} w(\cdot, t) d x=c$ for all $t$. Thus, $w$ is an FS of (1.6) with initial mass $c$. By uniqueness, $w=E_{c}$. Consequently, $v \leq \lim _{\tau \backslash 0} v_{\tau}=E_{c}$.

Let $u_{1}$ and $u_{2}$ be any two FS solutions of (1.1) with initial mass $c$. Then $u_{i} \leq E_{c}$ for $i=1,2$, so that by the contraction principle, for any $t>s>0$,

$$
\begin{aligned}
\int_{\mathbb{R}^{n}}\left|u_{1}(\cdot, t)-u_{2}(\cdot, t)\right| & \leq \int_{\mathbb{R}^{n}}\left|u_{1}(\cdot, s)-u_{2}(\cdot, s)\right| \\
& \leq \int_{\mathbb{R}^{n}}\left\{\left|u_{1}(\cdot, s)-E_{c}(\cdot, s)\right|+\left|E_{c}(\cdot, s)-u_{2}(\cdot, s)\right|\right\} \\
& =\int_{\mathbb{R}^{n}}\left\{\left[E_{c}(\cdot, s)-u_{2}(\cdot, s)\right]+\left[E_{c}(\cdot, s)-u_{2}(\cdot, s)\right]\right\} .
\end{aligned}
$$

Sending $s \searrow 0$ we conclude that $u_{1}(\cdot, t)=u_{2}(\cdot, t)$, since all the integrals $\int E_{c}(\cdot, s)$, $\int u_{1}(\cdot, s)$, and $\int u_{2}(\cdot, s)$ approach $c$ as $s \searrow 0$. This completes the proof of the theorem.

\subsection{Uniqueness of VSS for $u_{t}=\operatorname{div}\left(|\nabla u|^{p-2} \nabla u\right)-u^{q}$.}

Theorem 4.3. Assume (3.1). Then problem (1.1) has a unique VSS.

Proof. Let $u_{\infty}$ be the VSS established in Theorem 3.4. We first show that $u_{\infty}$ is the minimal singular VSS solution. Namely, any VSS of (1.1) is no less than $u_{\infty}$. Since $u_{\infty}$ is the limit of $u_{c}$, we need only show that any VSS of (1.1) is an upper bound of any FS of (1.1).

For this purpose, let $u$ be any VSS of (1.1). Let also $c>0$ be any fixed constant. Since $u$ satisfies (1.4), for all sufficiently small $\tau>0$, there exists a nonnegative continuous function $\varphi_{\tau}(\cdot)$ defined on $\mathbb{R}^{n}$ such that (i) $\varphi_{\tau}(\cdot) \leq u(\cdot, \tau)$ and (ii) $\int_{\mathbb{R}^{n}} \varphi_{\tau}(x) d x=c$. As $u(\cdot, \tau) \leq B \tau^{1 /(2-p)}|x|^{-p /(2-p)}$, we have

$$
\limsup _{\tau \searrow 0} \int_{|x|>\varepsilon} \varphi_{\tau}(x, \tau) d x \leq \limsup _{\tau \searrow 0} \int_{|x|>\varepsilon} B \tau^{1 /(2-p)}|x|^{-p /(2-p)} d x=0 \quad \forall \varepsilon>0 .
$$

Thus $\left\{\varphi_{\tau}\right\}_{\tau>0}$ is a $c \delta$-family. Consequently, from Theorem 3.1 and Theorem 4.2 , $\lim _{\tau \backslash 0} u^{\tau} \rightarrow u_{c}$ where $u^{\tau}$ is the solution of (1.1) with initial value $u^{\tau}(\cdot, 0)=\varphi_{\tau}$. Also by comparison, we have $u^{\tau}(\cdot, \cdot) \leq u(\cdot, \cdot+\tau)$ in $\mathbb{R}^{n} \times(0, \infty)$. It then follows that $u_{c}(\cdot, t) \leq u$. Thus, every VSS of (1.1) is an upper bound of every FS. Consequently, $u_{\infty}$ is the minimal VSS.

Next we show that $u_{\infty}$ is self-similar. Since $u_{c}$ is unique, it must be radially symmetric. As $u_{\infty}$ is the limit, as $c \rightarrow \infty$ of $u_{c}$, so is $u_{\infty}$ radially symmetric.

Now following the same proof for the self-similarity of $u^{*}$ in the proof of Lemma 2.3 we can show that $u_{\infty}$ is scaling invariant; namely, $u_{\infty}=\mathcal{T}^{\ell}\left(u_{\infty}\right)$ for every $\ell>0$. Thus $u_{\infty}$ is a self-similar solution of (1.1). Consequently, from Proposition 1, we see that $u^{*}=u_{\infty}$. As $u^{*}$ is the maximal VSS and $u_{\infty}$ the minimal VSS, we conclude that all VSS coincide with $u^{*}=u_{\infty}$. This completes the proof of the theorem. 
Note that our main Theorem 1.1 follows from Lemma2.1. Theorem 2.4 Corollary 3.3. and Theorems $3.4,4.2$, and 4.3

\section{REFERENCES}

[1] H. Brezis \& A. Friedman, Nonlinear parabolic equations involving measures as initial conditions, J. Math. Pures Appl., 62(1983), 73-97. MR700049 (84g:35093)

[2] H. Brezis, L. A. Peletier \& D. Terman, A very singular solution of the heat equation with absorption, Arch. Rational Mech. Anal. , 96(1985), 185-209.

[3] Xinfu Chen, Yuanwei Qi, \& Mingxin Wang, Self-similar very singular solutions of the parabolic p-Laplacian with absorption, J. Differential Equuations, 190 (2003), 1-15. MR/1970953 (2004c:34050)

[4] Xinfu Chen, Yuanwei Qi, \& Mingxin Wang, Classification of singular solutions of porous medium equations with absorption, Proc. Roy. Edinburgh, Ser. A, 135, 2005, 563-584. MR.2153436 (2006d:35125)

[5] J. I. Diaz \& J. E. Saa, Uniqueness of very singular self-similar solution of a quasilinear degenerate parabolic equation with absorption, Publ. Math., 36(1992), 19-38. MR.1179599 (93g:35079)

[6] E. DiBenedetto, Degenerate Parabolic Equations, Springer-Verlag, New York, 1993. MR1230384 (94h:35130)

[7] M. Escobedo, O. Kavian, \& H. Matano, Large time behavior of a dissipative semilinear heat equation, Comm. Partial Diff. Eqns., 20(1995), 1427-1452. MR.1335757 (96f:35074)

[8] V. A. Galaktionov, S. P. Kurdyumov, \& A. A. Samarskii, On asymptotic "eigenfunctions" of the Cauchy problem for a non-linear parabolic equation, Math. USSR Sbornik, 54(1986), 421-455.

[9] S. Kamin \& L. A. Peletier, Singular solutions of the heat equation with absorption, Proc. Amer. Math. Soc., 95(1985), 205-210. MR801324 (87b:35090)

[10] S. Kamin \& L.A. Peletier, Source-type solutions of degenerate diffusion equations with absorption, Israel J. Math., 50(1985), 219-230. MR793855 (87a:35112)

[11] S. Kamin, L. A. Peletier \& J. L. Vazquez, Classification of singular solutions of a nonlinear heat equation, Duke Math. J., 58(1989), 601-615. MR1016437 (91g:35137)

[12] S. Kamin \& J. L. Vazquez, Fundamental solutions and asymptotic behaviour for the $p$ Laplacian equation, Rev. Mat. Iberoamericana, 4(1988), 339-352. MR1028745 (90m:35020)

[13] S. Kamin \& J. L. Vazquez, Singular solutions of some nonlinear parabolic equations, J. Analyse Math., 59(1992), 51-74. MR1226951 (94e:35079)

[14] S. Kamin \& L. Veron, Existence and uniqueness of the very singular solution for the porous media equation with absorption, J. Analyse Math., 51(1988), 245-258. MR963156 (90f:35097)

[15] M. Kwak, A porous media equation with absorption. I. Long time behaviour, J. Math. Anal. Appl., 223(1998), 96-110. MR1627352(99e:35096a)

[16] M. Kwak, A porous media equation with absorption. II. Uniqueness of the very singular solution, J. Math. Anal. Appl., 223(1998), 111-125. MR1627348(99e:35096b)

[17] G. Leoni, A very singular solution for the porous media equation $u_{t}=\triangle\left(u^{m}\right)-u^{p}$ when $0<m<1$, J. Differential Equations, 132(1996), 353-376. MR1422124 (97j:35062)

[18] G. Leoni, On very singular self-similar solutions for the porous media equation with absorption, Differential and Integral Equations, 10(1997), 1123-1140. MR 1608045 (99e:35098)

[19] G. Leoni, Classification of positive solutions of the problem $\operatorname{div}\left(|\nabla u|^{p-2} \nabla u\right)+x \cdot \nabla u^{q}+\alpha u^{q}=$ 0 in $R^{n}$, Differ. Uravn. (Russian), 34(1998), 1170-1178; translation in Differential Equations, 34(1998), 1172-1180(1999). MR1693586 (2000b:35065)

[20] L. Oswald, Isolated, positive singularities for a nonlinear heat equation, Houston J. Math., 14(1988), 543-572. MR998457 (90k:35128)

[21] L. A. Peletier \& D. Terman, A very singular solution of the porous media equation with absorption, J. Differential Equations, 65(1986), 396-410. MR865069 (88b:35090)

[22] L. A. Peletier \& J. Wang, A very singular solution of a quasi-linear degenerate diffusion equation with absorption, Trans. Amer. Math. Soc., 307(1988), 813-826. MR940229 (89e:35081)

[23] L. A. Peletier \& J. Zhao, Source-type solutions of the porous media equation with absorption: The fast diffusion case, Nonlinear Analysis, TMA, 14(1990), 107-121. MR1036202 (91k:35140) 
[24] L. A. Peletier \& J. Zhao, Large time behaviour of solutions of the porous media equation with absorption: The fast diffusion case, Nonlinear Analysis, TMA, 17(1991), 991-1009. MR.1135955 (93d:76068)

Department of Mathematics, University of Pittsburgh, Pittsburgh, Pennsylvania 15260

E-mail address: xinfu@pitt.edu

Department of Mathematics, University of Central Florida, Orlando, Florida 32816

E-mail address: yqi@pegasus.cc.ucf.edu

Department of Applied Mathematics, Southeast University, Nanjing 210018, People's Republic of China

E-mail address: mxwang@seu.edu.cn 